\title{
AN EVAPORATION HEAT ENGINE AND CONDENSATION HEAT PUMP
}

\author{
N. G. BARTON ${ }^{1}$ \\ (Received 12 February, 2007; revised 26 February, 2008)
}

\begin{abstract}
This paper presents a thermodynamic model for a heat engine based on evaporative cooling of unsaturated air at reduced pressure. Also analysed is a related heat pump based on condensation of water vapour in moist air at reduced pressure. These devices operate as two-stroke reciprocating engines, which are their simplest possible embodiments. The mathematical models for the two devices are based on conservation of mass for both air and water vapour, ideal gas laws, constant specific heats, and, as appropriate, either constant entropy processes or cooling/heating by evaporation/condensation. Both models take the form of coupled algebraic systems in six variables, which require numerical solution for certain stages of the cycle. The specific work output of the heat engine increases as the inlet air becomes hotter and as the expansion ratio of the engine increases. The engine provides evaporative cooling of air from inlet to outlet. The heat pump has a good coefficient of performance, which decreases as the expansion ratio increases. The heat pump also has the effect of drying the air from inlet to outlet, producing distilled water as a by-product.
\end{abstract}

2000 Mathematics subject classification: 80-04, 80A20, 80A99.

Keywords and phrases: heat engine, heat pump, evaporation, condensation, low-pressure, thermodynamic cycles, ideal gases.

\section{Introduction}

The Barton Evaporation Engine (BEE) is a heat engine which exploits a low-pressure gas cycle as shown in Figure 1(a). Throughout, 'cycle' is used interchangeably to mean the operation of the engine in which air is taken in, processed and then vented, as well as a closed-loop cycle in which a fixed mass of gas undergoes heat transfer and change of volume and pressure.

The conventional Brayton cycle used in jet engines and gas turbines involves the steps compression-heating-expansion-exhaust. In contrast, operation of the BEE involves expansion of air taken into the engine, and then cooling at reduced pressure. The BEE cycle is completed by re-compression back to ambient pressure, and

\footnotetext{
${ }^{1}$ Sunoba Pty Ltd, P.O. Box 1295, Macquarie Centre, NSW 2113, Australia; e-mail: noel.barton@sunoba.com.au.

(C) Australian Mathematical Society 2008, Serial-fee code 0334-2700/08
} 


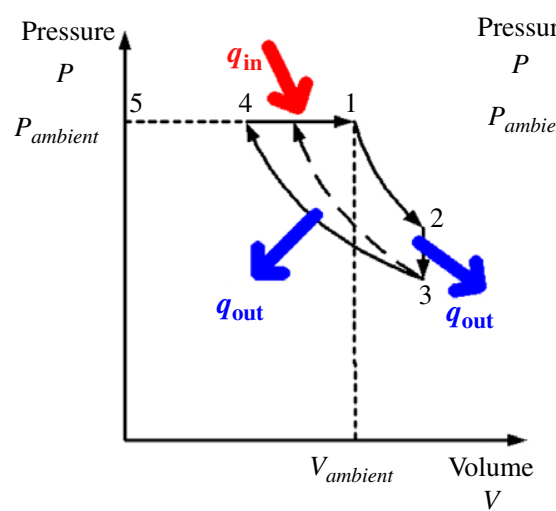

(a)

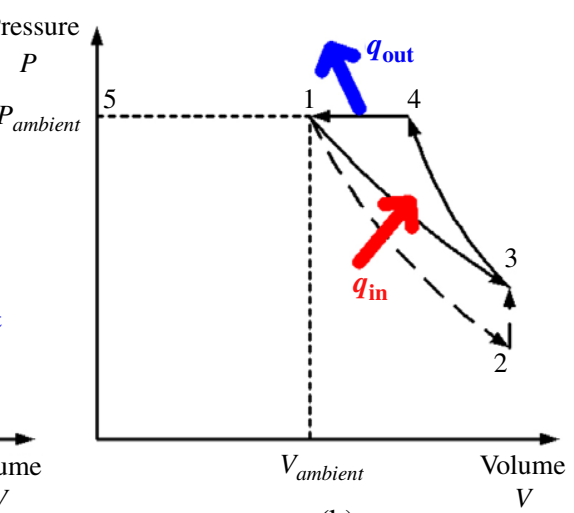

(b)

FIGURE 1. (a) Illustration of the thermodynamic cycle 1-2-3-4-5 for the BEE heat engine. Numbers refer to various stations as used in this paper. Heat is provided to the engine between stations 5 and 1 by the internal energy in the inlet air. Cooling between stations 2 and 3 and between 3 and 4 is accomplished by evaporation of a fog of water droplets sprayed into the low-pressure air. The dashed line from station 3 represents compression without further evaporation. (b) Illustration of the thermodynamic cycle 1-34-5 for the BDE heat pump. Heat is provided between stations 1 and 3 by latent heat of condensation. Heat transfer out of the BDE takes place during the exhaust. The dashed line between stations 1 and 2 represents expansion without condensation.

then exhaust. In the expansion stage, work must be done as the gas is expanded against atmospheric pressure. A greater amount of work is received during re-compression as the atmosphere does work on the gas.

In the BEE, the cycle 1-2-3-4-5 of Figure 1(a) is executed by a two-stroke pistonin-cylinder arrangement. This is the simplest manifestation of the thermodynamic cycle. There are other possibilities to execute the cycle, including the use of the Bernoulli effect for a compressible gas (see Barton [2]), and the use of a turbine and extraction fan in series. Cooling at constant volume between stations 2 and 3 is accomplished by spraying microscopic water droplets into the air at the point of maximum expansion. The present work extends an incomplete analysis of the BEE thermodynamic cycle by Barton [1] by incorporating evaporation during the compression stage 3-4.

The Barton Drying Engine (BDE) is a heat pump which is closely related to the BEE. It too exploits a low-pressure gas cycle; see Figure 1(b). Heating during the expansion stage is provided by condensation of water vapour, thereby releasing latent heat.

The features of the BEE thermodynamic cycle are as follows; numbers refer to the stations shown in Figure 1(a).

- $5 \rightarrow 1$ : The cycle starts with the chamber empty and the piston fully inserted into the cylinder. As the piston is withdrawn, unsaturated air naturally containing some water vapour is drawn through inlet valves into the chamber at ambient pressure.

- $1 \rightarrow 2$ : Valves are closed and the first stroke continues. Work is expended as the piston is withdrawn adiabatically against atmospheric pressure. The air-vapour 
mixture has constant entropy and remains unsaturated, even up to the point of maximum expansion (station 2).

- $2 \rightarrow 3$ : This stage takes place with the piston stationary, that is at constant volume. Water is sprayed into the chamber, creating a fog of microscopic water droplets. The gas is cooled at low pressure by evaporation until saturation. The pressure in the chamber decreases.

- $3 \rightarrow 4$ : The second stroke commences and the air-vapour-fog mixture is compressed as the piston is forced back into the chamber under the action of external pressure. Further evaporation occurs from microscopic droplets in the fog, and the entropy of the entire air-vapour-fog mixture is constant. Work is received during this stage.

- $4 \rightarrow$ 5: The second stroke is completed with the piston plunging fully into the chamber. The air-vapour-fog mixture is vented at ambient pressure through exhaust valves. The air is cooler and moister than at the inlet, and net work is obtained during the cycle. The BEE is a heat engine.

The BDE heat pump is closely related to the BEE heat engine. It operates on a similar two-stroke piston-in-cylinder arrangement, but there is a major difference in that saturation of the air-vapour mixture occurs during the expansion phase. Provided condensation nuclei are present, condensation of vapour will occur, liberating latent heat. Referring to Figure 1(b), the steps are as follows (note that station 2 is deliberately omitted from the discussion for consistency with the analysis of the BEE).

- $5 \rightarrow 1$ : The cycle starts with the chamber empty and the piston fully inserted into the cylinder. As the piston is withdrawn, moist air is drawn through inlet valves into the chamber at ambient pressure.

- $1 \rightarrow 3$ : Valves are closed and the first stroke continues. Work is expended as the piston is withdrawn adiabatically against atmospheric pressure. At some point during the expansion, the air-vapour mixture will become saturated and condensation will occur provided condensation nuclei are present. This causes a fog of microscopic water droplets to form. The entropy of the entire air-vapourfog mixture is constant during expansion. At the completion of the first stroke (station 3), the fog of condensed droplets is collected as chilled distilled water, leaving behind a saturated air-vapour mixture.

- $3 \rightarrow 4$ : The second stroke commences and the air-vapour mixture is compressed isentropically as the piston is forced back into the chamber under the action of external pressure. The temperature rises and the air-vapour mixture moves away from saturation. Work is received during this stage.

- $4 \rightarrow 5$ : The second stroke is completed with the piston plunging fully into the chamber. The air-vapour mixture is vented at ambient pressure through exhaust valves. The air is hotter and drier than at the inlet, and net work has been supplied to complete the cycle. The BDE is a heat pump. 
Thermodynamic models for the BEE and BDE are presented in Sections 2 and 4 respectively. The models take the form of coupled algebraic equations expressing familiar concepts such as conservation of mass of air and vapour, ideal gas laws, and, as appropriate, either constant entropy processes or cooling/heating by evaporation/condensation. Results from the models are provided in Sections 3 and 5 for the BEE and BDE respectively.

To highlight the results and overall theoretical conclusions, the work output from the BEE heat engine increases with the expansion ratio and the temperature of the inlet air. Illustrative results are now given for the case when the ambient air is at $35^{\circ} \mathrm{C}$ and relative humidity $35.5 \%$, the injected water has temperature $20^{\circ} \mathrm{C}$ and the backwork ratio (work expended: work received) is $80 \%$. Without pre-heating, the BEE heat engine functions as an evaporative cooler, which cools the air by $12.2^{\circ} \mathrm{C}$ at the same time as it delivers $346 \mathrm{~J} \mathrm{(kg} \mathrm{dry} \mathrm{air} \mathrm{throughput)}{ }^{-1}$. If the ambient air is heated to $100^{\circ} \mathrm{C}$ before being presented to the inlet valves, the specific work output is $7.2 \mathrm{~kJ}(\mathrm{~kg}$ dry air throughput $)^{-1}$ and the water consumption is $11.8 \mathrm{~kg} / \mathrm{kWh}$. If the ambient air is heated to $400{ }^{\circ} \mathrm{C}$, the specific work output is $106 \mathrm{~kJ}$ (kg dry air throughput) ${ }^{-1}$ and the water consumption is $3.4 \mathrm{~kg} / \mathrm{kWh}$.

The BDE heat pump has a good coefficient of performance, which decreases as the expansion ratio is increased. Chilled distilled water is produced as a by-product of BDE operation. The following illustrative results are given for the case when the temperature of the expanded air at Station 3 is just above freezing. If the inlet air is saturated and at $15^{\circ} \mathrm{C}, 5.1 \mathrm{~g}$ of water is condensed per kg of dry air, the specific work requirement is $509 \mathrm{~J}$ ( $\mathrm{kg}$ dry air throughput $)^{-1}$ and the exhaust temperature is $28.1^{\circ} \mathrm{C}$. If the inlet air is saturated and at $25^{\circ} \mathrm{C}, 12 \mathrm{~g}$ of water is condensed per $\mathrm{kg}$ of dry air, the specific work requirement is $2298 \mathrm{~J}$ (kg dry air throughput) ${ }^{-1}$ and the exhaust temperature is $56.6^{\circ} \mathrm{C}$.

It is expected that both the BEE heat engine and the BDE heat pump would handle a large volume of air. In their piston-in-cylinder form, these devices would be large, slow-revving and unobtrusive. Comments on possible applications of the devices are given in Section 6.

\section{Thermodynamic model for the BEE heat engine}

Between stations 1 and 2 of Figure 1(a), the air-vapour mixture in the chamber is expanded isentropically. The model equations are

$$
\begin{aligned}
\rho_{a 1} V_{1}=\rho_{a 2} V_{2}=m_{a} & \text { (mass of air) } \\
\rho_{v 1} V_{1}=\rho_{v 2} V_{2}=m_{v} & \text { (mass of vapour) } \\
\frac{P_{a 1}}{\rho_{a 1} T_{1}}=\frac{P_{a 2}}{\rho_{a 2} T_{2}}=R_{a} & \text { (ideal gas law for air) } \\
\frac{P_{v 1}}{\rho_{v 1} T_{1}}=\frac{P_{v 2}}{\rho_{v 2} T_{2}}=R_{v} & \text { (ideal gas law for vapour) }
\end{aligned}
$$




$$
\begin{gathered}
m_{a}\left[s_{a 0}+C_{a V} \log \frac{P_{a 1}}{\rho_{a 1}^{\gamma_{a}}}\right]+m_{v}\left[s_{v 0}+C_{v V} \log \frac{P_{v 1}}{\rho_{v 1}^{\gamma_{v}}}\right] \\
=m_{a}\left[s_{a 0}+C_{a V} \log \frac{P_{a 2}}{\rho_{a 2}^{\gamma_{a}}}\right]+m_{v}\left[s_{v 0}+C_{v V} \log \frac{P_{v 2}}{\rho_{v 2}^{\gamma_{v}}}\right], \\
\frac{V_{2}}{V_{1}}=r .
\end{gathered}
$$

In equations (2.1)-(2.6) and throughout the paper, $P, \rho, s, T$ and $V$ denote pressure, density, specific entropy, temperature and volume respectively. Other constants include the specific heat capacity $C$ (assumed constant), mass of gas $m$, ideal gas constant $R$, and the expansion ratio $r$. Subscripts $a$ and $v$ denote air and vapour, numerical subscripts 1-5 refer to the stations shown in Figure 1(a), numerical subscript 0 refers to arbitrary constants, and subscripts $P$ and $V$ refer to processes at constant pressure and volume respectively. The ratio of specific heats is $\gamma=C_{P} / C_{V}$. Equation (2.5) (see for example [6, Section 5.4] or [3, Section 1.7]) states that the total entropy of the air-vapour mixture is constant during the expansion stage, which requires that no heat be added between stations 1 and 2 and that the process is reversible.

A brief discussion is warranted as to whether water vapour can be represented by an ideal gas with constant specific heats. Çengel and Boles [4, Figure 2-48] show the relative error when the specific volume is calculated using ideal gas theory. For vapour pressures less than $100 \mathrm{kPa}$, the relative error is less than $1.6 \%$, even near saturation. Çengel and Boles [4, Figure 3-76] show the temperature dependence of specific heat capacities at constant pressure. For both air and water, the variation is modest provided the temperature is less than $500^{\circ} \mathrm{C}$. Provided temperatures do not exceed $500^{\circ} \mathrm{C}$ and pressures are less than atmospheric, it is reasonable to assume that both air and vapour are ideal gases with constant specific heat capacities.

On elimination of arbitrary constants, (2.5) gives

$$
\left(\frac{P_{a 1}}{\rho_{a 1}^{\gamma_{a}}}\right)^{m_{a} C_{a V}}\left(\frac{P_{v 1}}{\rho_{v 1}^{\gamma_{v}}}\right)^{m_{v} C_{v V}}=\left(\frac{P_{a 2}}{\rho_{a 2}^{\gamma_{a}}}\right)^{m_{a} C_{a V}}\left(\frac{P_{v 2}}{\rho_{v 2}^{\gamma_{v}}}\right)^{m_{v} C_{v V}} .
$$

Since (2.1), (2.2) and (2.3) give $\rho_{a 2}=\rho_{a 1} / r$ and $\rho_{v 2}=\rho_{v 1} / r,(2.7)$ becomes

$$
\left(\frac{P_{a 1}}{P_{a 2}}\right)^{m_{a} C_{a V}}\left(\frac{P_{v 1}}{P_{v 2}}\right)^{m_{v} C_{v V}}=r^{\gamma_{a} m_{a} C_{a V}+\gamma_{v} m_{v} C_{v V}} .
$$

From the equations of state (2.3) and (2.4), the partial pressures $P_{a 2}$ and $P_{v 2}$ can be expressed in terms of the temperatures $T_{1}$ and $T_{2}$ as follows: $P_{a 2}=P_{a 1} T_{2} /\left(r T_{1}\right)$ and $P_{v 2}=P_{v 1} T_{2} /\left(r T_{1}\right)$. Equation (2.8) thus gives

$$
T_{2}=T_{1} r^{-d_{1} / d_{2}}
$$


in which $d_{1}$ and $d_{2}$ are defined by

$$
\begin{aligned}
& d_{1}=\left(\gamma_{a}-1\right) m_{a} C_{a V}+\left(\gamma_{v}-1\right) m_{v} C_{v V}, \\
& d_{2}=m_{a} C_{a V}+m_{v} C_{v V} .
\end{aligned}
$$

Once $T_{2}$ has been calculated, all other properties at station 2 can be calculated by direct substitution into equations above, thereby completing the model for the transition from station 1 to station 2 .

Between stations 2 and 3, suppose that mass $\delta m_{v}$ of water is sprayed into the chamber at temperature $T_{w i}$ and evaporates at constant volume. The subscript $w i$ denotes injected water and the volume of water is neglected. The equations for the transition from station 2 to station 3 are

$$
\begin{array}{rlrl}
\rho_{a 2} V_{2} & =\rho_{a 3} V_{3} & & \text { (mass of air) }, \\
\rho_{v 2} V_{2}+\delta m_{v} & =\rho_{v 3} V_{3} & & \text { (mass of water) } \\
\frac{P_{a 2}}{\rho_{a 2} T_{2}} & =\frac{P_{a 3}}{\rho_{a 3} T_{3}}=R_{a} & & \text { (ideal gas law for air), } \\
\frac{P_{v 2}}{\rho_{v 2} T_{2}} & =\frac{P_{v 3}}{\rho_{v 3} T_{3}}=R_{v} & & \text { (ideal gas law for vapour) } \\
m_{a} u_{a 2}+m_{v} u_{v 2}+\delta m_{v} u_{w i} & =m_{a} u_{a 3}+\left(m_{v}+\delta m_{v}\right) u_{v 3}, \\
V_{2} & =V_{3} .
\end{array}
$$

Here $u$ is the specific internal energy and equation (2.16) states that the total internal energy of the air-vapour-water mixture is constant during the evaporation process, which takes place with the chamber volume held fixed. Because the specific heats have been assumed constant, the specific internal energy terms in (2.16) can be written as

$$
\begin{aligned}
& u_{a 2}=u_{a 0}+C_{a V}\left(T_{2}-T_{0}\right), \\
& u_{a 3}=u_{a 0}+C_{a V}\left(T_{3}-T_{0}\right), \\
& u_{v 2}=u_{v 0}+C_{v V}\left(T_{2}-T_{0}\right), \\
& u_{v 3}=u_{v 0}+C_{v V}\left(T_{3}-T_{0}\right), \\
& u_{w i}=u_{v 2}-C_{v V}\left(T_{2}-T_{w i}\right)-L\left(T_{w i}\right) .
\end{aligned}
$$

In the last of these equations, the three terms are respectively the internal energy of vapour at $T_{2}$, the sensible heat adjustment required to give the internal energy of vapour at $T_{w i}$, and the latent heat of evaporation of water at temperature $T_{w i}$. Taking account of these expressions for the specific internal energy, equation (2.16) becomes

$$
T_{3}=T_{2}-\frac{\delta m_{v}\left[C_{v V}\left(T_{2}-T_{w i}\right)+L\left(T_{w i}\right)\right]}{d_{3}},
$$

in which $d_{3}$ is defined by

$$
d_{3}=m_{a} C_{a V}+\left(m_{v}+\delta m_{v}\right) C_{v V} .
$$

Once $T_{3}$ has been thus calculated, all other properties at station 3 follow by substitution. 
It remains to calculate the amount of water evaporated, $\delta m_{v}$. This is done iteratively as follows. From the foregoing analysis based on an assumed value of $\delta m_{v}$, the temperature $T_{3}$ and vapour pressure $P_{v 3}$ can be calculated. However, at a particular temperature $T_{3}$, saturation occurs when $P_{v 3}$ is equal to the saturation pressure given by the Clapeyron-Clausius equation [4, Section 11-3]. If $P_{v 3}$ is above the saturation vapour pressure, then $\delta m_{v}$ is reduced; if $P_{v 3}$ is below the saturation vapour pressure, then $\delta m_{v}$ is increased. A simple iterative loop is applied until $P_{v 3}$ is sufficiently close to the saturation vapour pressure, thereby completing the model for the transition from station 2 to station 3 .

Re-compression from station 3 to station 4 is now discussed. It is first assumed that further evaporation does not occur; subsequently the analysis will be modified to allow for further evaporation.

The model equations for re-compression without evaporation follow the development of Barton [1], and are similar to those for the transition from station 1 to station 2:

$$
\begin{array}{cl}
\rho_{a 3} V_{3}=\rho_{a 4} V_{4}=m_{a} & \text { (mass of air), } \\
\rho_{v 3} V_{3}=\rho_{v 4} V_{4}=m_{v}+\delta m_{v} & \text { (mass of vapour), } \\
\frac{P_{a 3}}{\rho_{a 3} T_{3}}=\frac{P_{a 4}}{\rho_{a 4} T_{4}}=R_{a} & \text { (ideal gas law for air), } \\
\frac{P_{v 3}}{\rho_{v 3} T_{3}}=\frac{P_{v 4}}{\rho_{v 4} T_{4}}=R_{v} & \text { (ideal gas law for vapour), } \\
m_{a}\left[s_{a 0}+C_{a V} \log \frac{P_{a 3}}{\rho_{a 3}^{\gamma_{a}}}\right]+\left(m_{v}+\delta m_{v}\right)\left[s_{v 0}+C_{v V} \log \frac{P_{v 3}}{\rho_{v 3}^{\gamma_{v}}}\right] & \\
=m_{a}\left[s_{a 0}+C_{a V} \log \frac{P_{a 4}}{\rho_{a 4}^{\gamma_{a}}}\right]+\left(m_{v}+\delta m_{v}\right)\left[s_{v 0}+C_{v V} \log \frac{P_{v 4}}{\rho_{v 4}^{\gamma_{v}}}\right], \\
\frac{V_{4}}{V_{3}}=\theta .
\end{array}
$$

The volume ratio $\theta$ is unknown at this stage, but is eventually determined by matching the outlet pressure to the inlet pressure.

On elimination of arbitrary constants, (2.24) gives

$$
\left(\frac{P_{a 3}}{\rho_{a 3}^{\gamma_{a}}}\right)^{m_{a} C_{a V}}\left(\frac{P_{v 3}}{\rho_{v 3}^{\gamma_{v}}}\right)^{\left(m_{v}+\delta m_{v}\right) C_{v V}}=\left(\frac{P_{a 4}}{\rho_{a 4}^{\gamma_{a}}}\right)^{m_{a} C_{a V}}\left(\frac{P_{v 4}}{\rho_{v 4}^{\gamma_{v}}}\right)^{\left(m_{v}+\delta m_{v}\right) C_{v V}}
$$

Equations (2.20), (2.21) and (2.25) give $\rho_{a 4}=\rho_{a 3} / \theta$ and $\rho_{v 4}=\rho_{v 3} / \theta$, so (2.26) becomes

$$
\left(\frac{P_{a 3}}{P_{a 4}}\right)^{m_{a} C_{a V}}\left(\frac{P_{v 3}}{P_{v 4}}\right)^{\left(m_{v}+\delta m_{v}\right) C_{v V}}=\theta^{\gamma_{a} m_{a} C_{a V}+\gamma_{v}\left(m_{v}+\delta m_{v}\right) C_{v V}} .
$$


From the ideal gas laws (2.22) and (2.23), the partial pressures $P_{a 4}$ and $P_{v 4}$ can be eliminated in favour of the temperature $T_{4}: P_{a 4}=P_{a 3} T_{4} /\left(\theta T_{3}\right), P_{v 4}=P_{v 3} T_{4} /\left(\theta T_{3}\right)$. Equation (2.27) thus reduces to

$$
T_{4}=T_{3} \theta^{-d_{4} / d_{5}}
$$

in which $d_{4}$ and $d_{5}$ are defined by

$$
\begin{aligned}
& d_{4}=\left(\gamma_{a}-1\right) m_{a} C_{a V}+\left(\gamma_{v}-1\right)\left(m_{v}+\delta m_{v}\right) C_{v V}, \\
& d_{5}=m_{a} C_{a V}+\left(m_{v}+\delta m_{v}\right) C_{v V} .
\end{aligned}
$$

The volume ratio $\theta$ is determined by equating the outlet pressure to the inlet pressure. The outlet pressure is

$$
P_{a 4}+P_{v 4}=\left(P_{a 3}+P_{v 3}\right) \theta^{-\left(d_{4}+d_{5}\right) / d_{5}}
$$

and, since $P_{a 4}+P_{v 4}=P_{a 1}+P_{v 1}, \theta$ is found to be

$$
\theta=\left(\frac{P_{a 3}+P_{v 3}}{P_{a 1}+P_{v 1}}\right)^{d_{5} /\left(d_{4}+d_{5}\right)} .
$$

In the absence of evaporation, all quantities at station 4 can now be determined by substitution.

Suppose, however, that some water droplets at temperature $T_{w i}$ are present during compression, which is assumed to be adiabatic (i.e. no external heat transfer occurs). For example, these droplets might be injected once saturation has been reached at stage 3. After a short period of compression, conditions in the chamber will be such that the vapour pressure $P_{v}$ is below the saturation vapour pressure for the given temperature, and evaporation will occur. Moreover, such evaporation is reversible - the entropy of the entire air-vapour-fog mixture will be constant—with the sensible heat effects of the droplets playing a relatively unimportant role. That suggests that re-compression with evaporation can be calculated as a sequence of small steps, each involving two sub-steps:

(1) compression without evaporation as per equations (2.20)-(2.30), with constant entropy of the air-vapour component of the air-vapour-fog mixture (here the presence of droplets is ignored); and

(2) evaporative cooling to saturation at constant volume as per equations (2.12) (2.19) for the transition from station 2 to station 3.

For this compression model to be valid, changes in the entropy of the droplets should be negligible in sub-step 1, and changes in the internal energy of the droplets should be negligible compared to the latent heat of evaporation in sub-step 2. This will be the case when (a) the temperature during re-compression does not differ too much from the temperature $T_{w i}$ of surplus droplets, which is assumed not to change, or (b) the droplets are injected as required through the compression process. It is noted that the mass of vapour $m_{v}$ needs to be updated through the process, which means that constants $d_{3}, d_{4}$ and $d_{5}$ also need to be updated. 


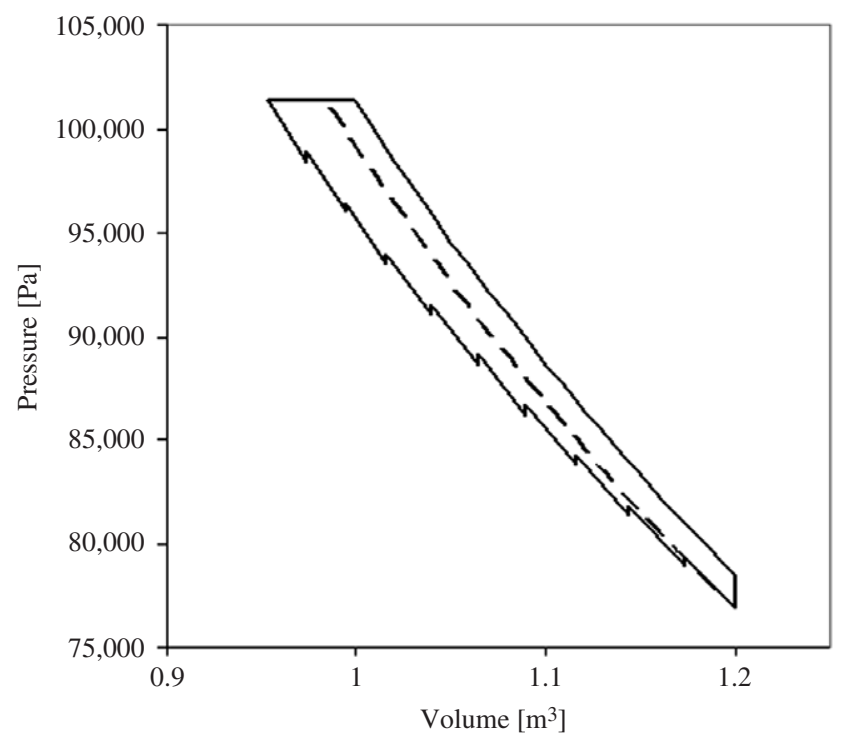

Figure 2. Simulations for the case $T_{1}=45^{\circ} \mathrm{C}, P_{a 1}=99000 \mathrm{~Pa}, P_{v 1}=2000 \mathrm{~Pa}, r=1.2, T_{w i}=20^{\circ} \mathrm{C}$. The dashed line represents re-compression without evaporation. The sawtooth line shows re-compression with evaporation (only 10 steps are shown above, but 60 steps were used for the results of Section 3). When evaporation occurs during compression, the net work output is more than twice that for the case when evaporation does not occur during compression.

This sequential approach is illustrated in Figure 2. The sawtooth line is an approximation to the state path on which the vapour pressure $P_{v}$ is always equal to the saturation vapour pressure given by the Clapeyron-Clausius law [4, Section 11-3].

To sum up, compression with evaporation is analysed by decomposing the transition into $N$ steps, each of which involves an increment to the pressure of

$$
\delta P=\frac{\left(P_{a 1}+P_{v 1}\right)-\left(P_{a 3}+P_{v 3}\right)}{N} .
$$

Each step has two sub-steps, the first described by appropriate modifications to equations (2.20)-(2.30), and the second by appropriate modifications to equations (2.12)-(2.19) together with iteration to determine the amount of evaporation at each step. An overall check on the calculation is that the entropy of the entire air-vapourfog mixture must be approximately constant from station 3 to station 4 .

The work output $W$ of the BEE is given by

$$
W=\int_{V_{1}}^{V_{2}}\left(P_{a}+P_{v}-\left[P_{a 1}+P_{v 1}\right]\right) d V+\int_{V_{3}}^{V_{4}}\left(P_{a}+P_{v}-\left[P_{a 1}+P_{v 1}\right]\right) d V .
$$

Work is neither expended nor received when inlet or outlet valves are open (i.e. disregarding any work required for intake and exhaust), nor during evaporative cooling 
TABLE 1. State of the engine for the entropy check. The amount of water evaporated during re-compression is $0.03916-0.02291=0.01625 \mathrm{~kg}$.

\begin{tabular}{ccccccc}
\hline Station & $V\left(\mathrm{~m}^{3}\right)$ & $T\left({ }^{\circ} \mathrm{C}\right)$ & $m_{a}(\mathrm{~kg})$ & $m_{v}(\mathrm{~kg})$ & $P_{a}(\mathrm{~Pa})$ & $P_{v}(\mathrm{~Pa})$ \\
\hline 1 & 1.0 & 130 & 0.85822 & 0.01075 & 99300 & 2000 \\
2 & 1.6 & 61.2 & 0.85822 & 0.01075 & 51475 & 1037 \\
3 & 1.6 & 16.6 & 0.85822 & 0.02291 & 44614 & 1915 \\
4 & 0.8158 & 39.4 & 0.85822 & 0.03916 & 94376 & 6924 \\
\hline
\end{tabular}

with the piston held in a fixed position. The first integral in (2.32) is negative and represents the work required for expansion, and the second integral is positive and is the work received during compression. For the expansion phase, equations (2.6) and (2.9) show that

$$
P_{a}+P_{v}=\left(P_{a 1}+P_{v 1}\right)\left(\frac{V}{V_{1}}\right)^{-\left(d_{1}+d_{2}\right) / d_{2}},
$$

which enables an analytical evaluation of the first integral. For the compression phase, $P$ and $V$ are known at the beginning and end of each of the sub-steps, and so the second integral can be evaluated numerically by the trapezoidal rule.

\section{Results for the BEE heat engine}

Details of computational procedures and checks are mentioned before presentation of results. Numerical tests showed that accurate results could be obtained using 60 steps for the transition from station 3 to station 4 , as described near the end of Section 2. A key requirement in the simulations was to determine saturation conditions for air-vapour mixtures. This is required both for the transition between stations 2 and 3 and each of the sub-steps 2 in the transition from station 3 to station 4 . At a particular temperature, saturation occurs when the partial vapour pressure $P_{v}$ reaches the saturation pressure given by the Clapeyron-Clausius equation [4, Section 11-3]. For the simulations presented below, the saturation vapour pressure was given by linear interpolation of tabulated values [4, Table A-4].

The entropy check anticipated near the end of Section 2 is now described for the case $V_{1}=1 \mathrm{~m}^{3}, T_{1}=130{ }^{\circ} \mathrm{C}, P_{a 1}=99300 \mathrm{~Pa}, P_{v 1}=2000 \mathrm{~Pa}, T_{w i}=20^{\circ} \mathrm{C}$ and $r=$ 1.6. These conditions (except for the volume) correspond to design specifications for an experimental BEE currently under construction.

Based on linear interpolation of Table 1 for specific entropy for air and saturated water and vapour [4, Tables A-4 and A-17], the change in entropy of the vapour-fog component of the air-vapour-fog mixture between stations 3 and 4 is $+118.61 \mathrm{~J} \mathrm{~K}^{-1}$, whilst the change in entropy of the air component of the mixture is $-119.34 \mathrm{~J} \mathrm{~K}^{-1}$. (Note that the specific entropy of air as an ideal gas depends on both temperature and pressure; see [4, Equation (6-42)].) The entropy change of the entire mixture is $-0.73 \mathrm{~J} \mathrm{~K}^{-1}$, which is acceptably close to zero given the various approximations made (constant specific heats, ideal gas laws, finite number of compression steps, 

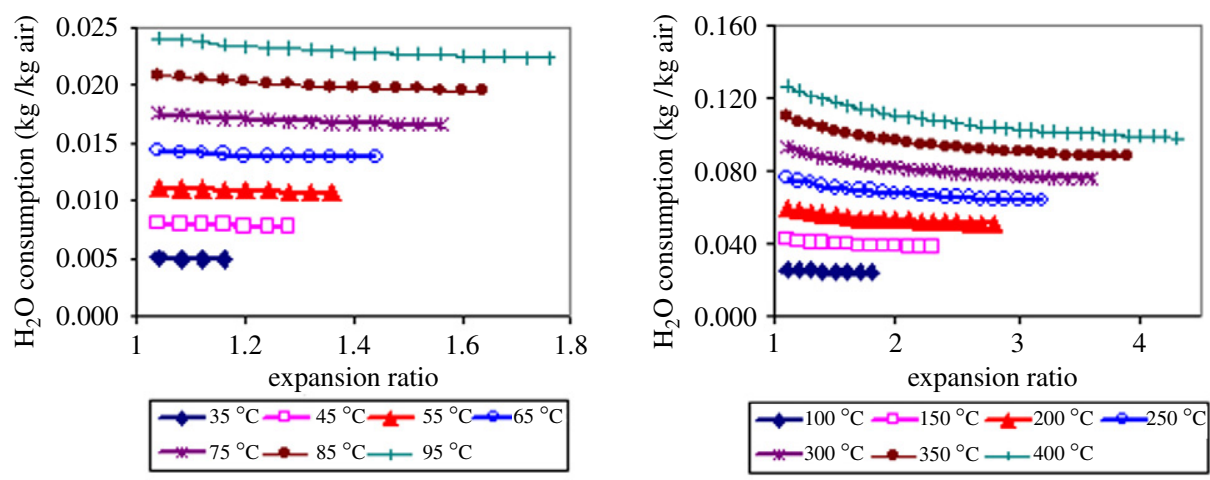

FIGURE 3. Mass of water $\delta m_{v}(\mathrm{~kg})$ evaporated per kg of dry air for both sets of inlet temperatures. Results are shown as a function of the expansion ratio $r$ for various values of inlet temperature $T_{1}$.

linear interpolation of various quantities). The magnitude of the entropy change, given by addition of two large quantities of opposing sign, is 0.003 times the sum of the magnitudes of the individual quantities. This gives confidence in the computational procedure described at the end of Section 2.

In the results presented in the remainder of this section, the ambient air was assumed to be at $35^{\circ} \mathrm{C}$ with partial pressures $P_{a 1}=99300 \mathrm{~Pa}$ and $P_{v 1}=2000 \mathrm{~Pa}$. This corresponds to a relative humidity of $35.5 \%$. The initial volume was $V_{1}=1 \mathrm{~m}^{3}$ and the temperature of the injected water was $20^{\circ} \mathrm{C}$.

The prime objective was to explore the sensitivity of the results to pre-heating of the air. Results are presented for a set of relatively low inlet temperatures $\left(T_{1}=35, \ldots, 95^{\circ} \mathrm{C}\right)$ and a second set with relatively high inlet temperatures $\left(T_{1}=\right.$ $\left.100, \ldots, 400{ }^{\circ} \mathrm{C}\right)$. The inlet densities of air and vapour were given by the ideal gas law $P=R \rho T$, where $R_{a}=287.0 \mathrm{~J}(\mathrm{~kg} \mathrm{~K})^{-1}$ and $R_{v}=461.5 \mathrm{~J}(\mathrm{~kg} \mathrm{~K})^{-1}$. The specific heat capacities (with units $\mathrm{J}(\mathrm{kg} \mathrm{K})^{-1}$ ) were fixed at the values of $20^{\circ} \mathrm{C}: C_{a P}=1005$, $C_{a V}=718, C_{v P}=1872, C_{v V}=1411$. The latent heat of evaporation, $L$, was given by linear interpolation of tabulated values [4, Table A-4]. An approximate value for $L$ is $2.3 \mathrm{MJ} \mathrm{kg}^{-1}$, which is slightly less than one-tenth the energy density of hydrocarbon fuels, without need for oxidant.

Figures 3 and 4 show the specific water consumption, $\delta m_{v} / m_{a}(\mathrm{~kg}(\mathrm{~kg}$ air throughput $)^{-1}$ ), and the specific work output for one cycle of the BEE heat engine, $w=W / m_{a}\left(\mathrm{~J} \mathrm{~kg}^{-1}\right)$. As the inlet temperature is increased, more water can be evaporated and the work output of the engine increases. The work output of the engine also increases as the expansion ratio $r$ increases, but only up to the point where saturation would be reached at station 2 .

Figures 5 and 6 show the back-work ratio and the temperature decrease from inlet to outlet. The back-work ratio is the work required for expansion divided by the work received during compression. In common with all gas cycle engines, the BEE has a high back-work ratio; values of perhaps $70 \%$ or more must be 

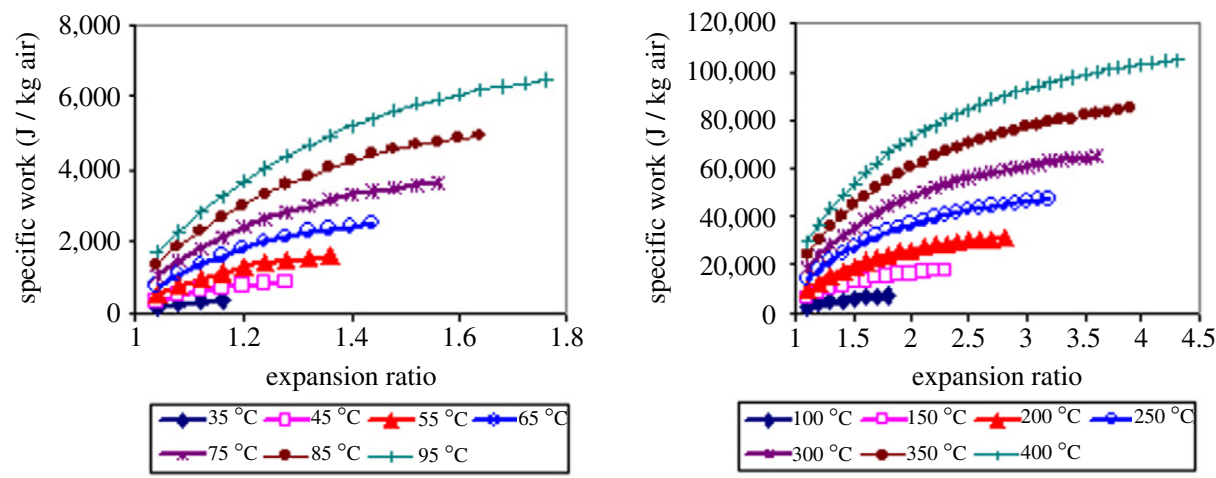

FIGURE 4. Specific work output $w\left(\mathrm{~J} \mathrm{~kg}^{-1}\right)$ for both sets of inlet temperatures. Results are shown as a function of the expansion ratio $r$ for various values of inlet temperature $T_{1}$.
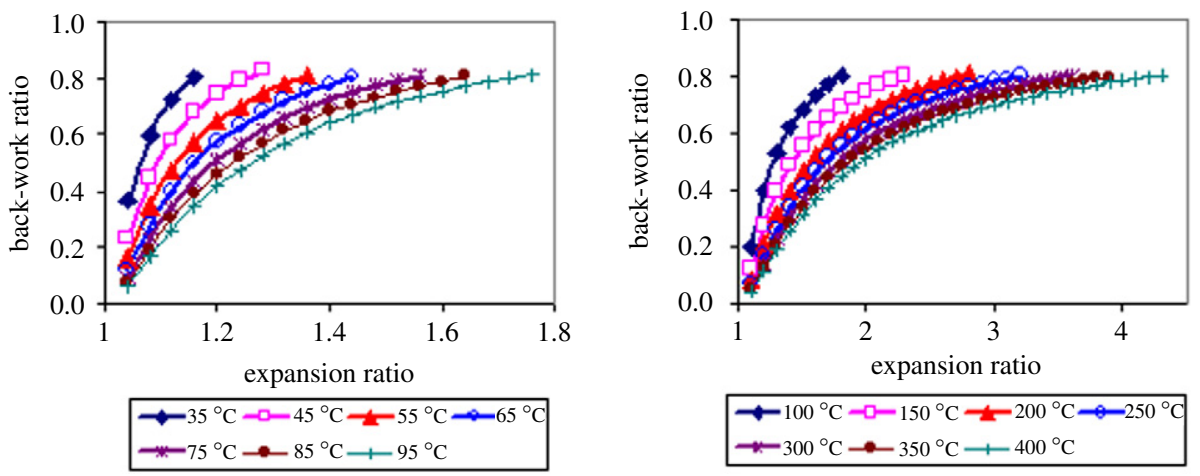

FIGURE 5. Back-work ratio (expansion work divided by compression work) for both sets of inlet temperatures. Results are shown as a function of the expansion ratio $r$ for various values of inlet temperature $T_{1}$.

considered as a design requirement. The temperature decrease from inlet to outlet shows the remarkable fact that evaporative coolers can also simultaneously deliver useful work output.

Efficiency is defined as desired output divided by required input. The desired output is the work per cycle, $W$, and the required input is the energy needed to pre-heat air from ambient temperature $T_{\mathrm{amb}}$ to the inlet temperature $T_{1}$. Thus the efficiency, $\eta$, is

$$
\eta=\frac{W}{\left(m_{a} C_{a P}+m_{v} C_{v P}\right)\left(T_{1}-T_{\mathrm{amb}}\right)} .
$$

Figure 7 shows the efficiency, which increases with $T_{1}$ and the expansion ratio. The left-hand figure does not include results for $T_{1}=35^{\circ} \mathrm{C}$, which is in fact the ambient temperature. At ambient temperature, the BEE heat engine delivers surplus work without any need for pre-heating. The engine is able to take energy from the air, 

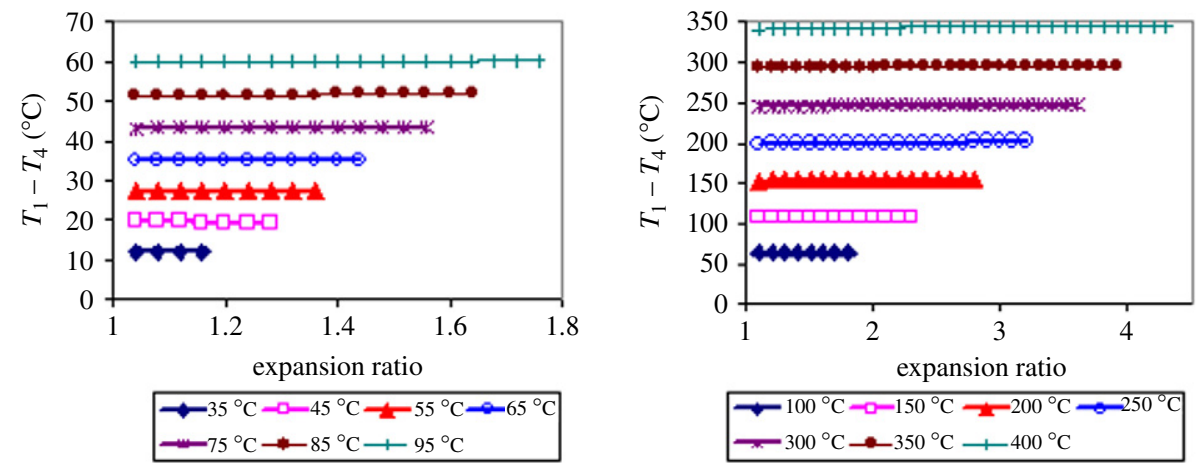

FIgURE 6. Temperature decrease $T_{1}-T_{4}\left({ }^{\circ} \mathrm{C}\right)$ between inlet and outlet for both sets of inlet temperatures. Results are shown as a function of the expansion ratio $r$ for various values of inlet temperature $T_{1}$.
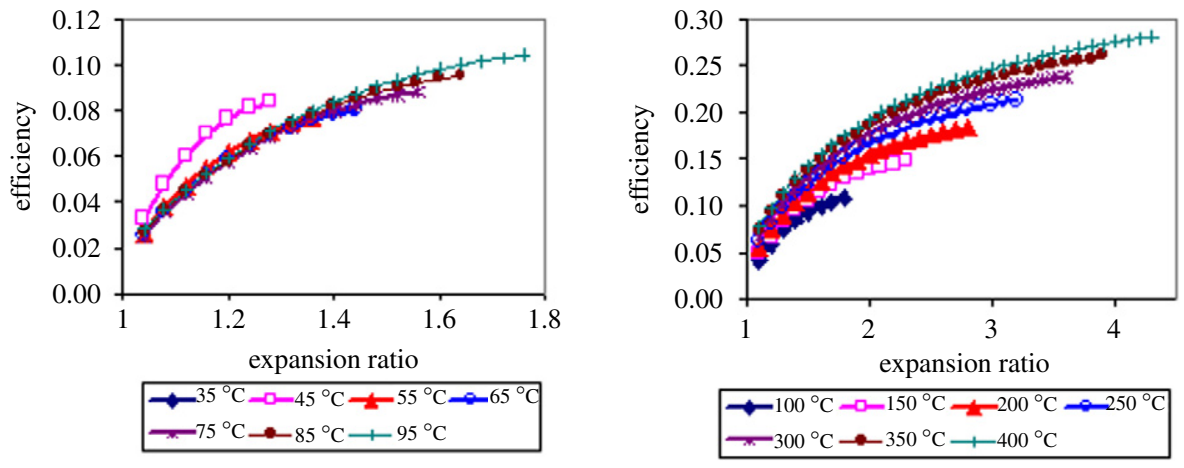

FIGURE 7. Efficiency $\eta$ as defined by equation (3.1) for both sets of inlet temperatures. Results are shown as a function of the expansion ratio $r$ for various values of inlet temperature $T_{1}$.
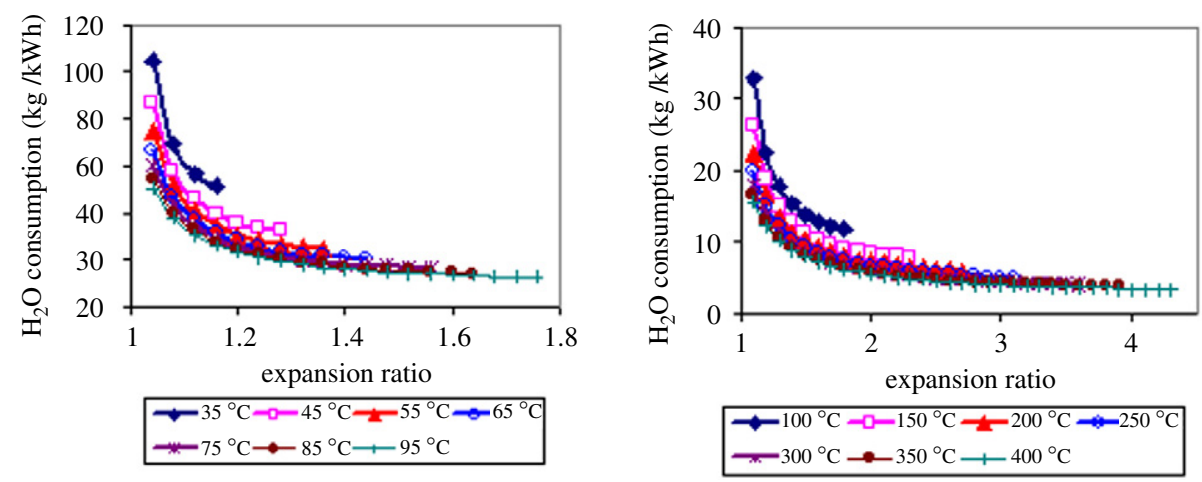

FIGURE 8. Water consumption expressed in $\mathrm{kg} / \mathrm{kWh}$ for both sets of inlet temperatures. Results are shown as a function of the expansion ratio $r$ for various values of inlet temperature $T_{1}$. 
thereby providing a cooling effect from inlet to exhaust, without violating the laws of thermodynamics. The increase in entropy due to evaporation offsets the decrease in entropy due to cooling. The efficiency is also remarkably high for inlet temperature $T_{1}=45^{\circ} \mathrm{C}$ since relatively little pre-heating is required.

Figure 8 shows the water consumption of the BEE expressed in $\mathrm{kg}$ of water per $\mathrm{kWh}$ of work produced. Water consumption is of great practical importance, since in general the injected water will need to be pure, thereby necessitating a water treatment plant as an auxiliary system. The water consumption drops rapidly with increasing inlet temperature, from approximately $51 \mathrm{~kg} / \mathrm{kWh}$ at $T_{1}=35^{\circ} \mathrm{C}$ and $80 \%$ back-work ratio to $3.4 \mathrm{~kg} / \mathrm{kWh}$ at $T_{1}=400{ }^{\circ} \mathrm{C}$ and $80 \%$ back-work ratio.

\section{Thermodynamic model for the BDE heat pump}

Model equations are now presented for the BDE heat pump, for which the thermodynamic cycle is illustrated in Figure 1(b). In general, it is assumed that the inlet air is saturated. Provided condensation nuclei (i.e. seed droplets) are present, the expansion phase from station 1 to station 3 will involve condensation. This is analysed using an approach similar to that presented at the end of Section 2 for the BEE heat engine. The starting point for the model equations is to assume that condensation does not occur between stations 1 and 2 as shown on Figure 1(b), only between stations 2 and 3. The two stages will then be combined into a multi-step transition from station 1 to station 3 .

Between stations 1 and 2 of Figure 1(b), suppose the air-vapour mixture is expanded adiabatically without condensation. This results in a set of equations identical to (2.1)-(2.6). The method of solution is the same as introduced in Section 2. The temperature $T_{2}$ is given by

$$
T_{2}=T_{1} r^{-e_{1} / e_{2}}
$$

in which $e_{1}$ and $e_{2}$ are defined by

$$
\begin{aligned}
& e_{1}=\left(\gamma_{a}-1\right) m_{a} C_{a V}+\left(\gamma_{v}-1\right) m_{v} C_{v V}, \\
& e_{2}=m_{a} C_{a V}+m_{v} C_{v V} .
\end{aligned}
$$

Once $T_{2}$ has been calculated, all other properties at station 2 can be calculated by direct substitution.

Between stations 2 and 3, suppose that the mass of vapour changes by $\delta m_{v}$; in fact condensation occurs, $\delta m_{v}<0$. The model equations are similar to the set (2.12)(2.17), but with changes to equation (2.16): 


$$
\begin{array}{rlrl}
\rho_{a 2} V_{2} & =\rho_{a 3} V_{3} & & \text { (mass of air) }, \\
\rho_{v 2} V_{2}+\delta m_{v} & =\rho_{v 3} V_{3} & & \text { (mass of water) }, \\
\frac{P_{a 2}}{\rho_{a 2} T_{2}} & =\frac{P_{a 3}}{\rho_{a 3} T_{3}}=R_{a} & & \text { (ideal gas law for air), } \\
\frac{P_{v 2}}{\rho_{v 2} T_{2}} & =\frac{P_{v 3}}{\rho_{v 3} T_{3}}=R_{v} \quad & \text { (ideal gas law for vapour) }, \\
m_{a} u_{a 2}+m_{v} u_{v 2} & =m_{a} u_{a 3}+\left(m_{v}+\delta m_{v}\right) u_{v 3}-\delta m_{v} u_{w 3}, \\
V_{2}=V_{3} . &
\end{array}
$$

The specific internal energy terms in (4.8) can be written as

$$
\begin{aligned}
u_{a 2} & =u_{a 0}+C_{a V}\left(T_{2}-T_{0}\right), \\
u_{a 3} & =u_{a 0}+C_{a V}\left(T_{3}-T_{0}\right), \\
u_{v 2} & =u_{v 0}+C_{v V}\left(T_{2}-T_{0}\right), \\
u_{v 3} & =u_{v 0}+C_{v V}\left(T_{3}-T_{0}\right), \\
u_{w 3} & =u_{v 3}-L\left(T_{3}\right),
\end{aligned}
$$

where $L\left(T_{3}\right)$ is the latent heat of condensation at temperature $T_{3}$ with the volume held constant, $V_{3}=V_{2}$. (Implicit in the last equation is an assumption that condensation is occurring under saturated conditions for both liquid water and vapour.) It follows that $T_{3}$ is given by

$$
T_{3}=T_{2}-\frac{\delta m_{v} L\left(T_{3}\right)}{e_{3}} \quad\left(\delta m_{v}<0\right),
$$

in which $e_{3}$ is defined by

$$
e_{3}=m_{a} C_{a V}+m_{v} C_{v V} .
$$

Once $T_{3}$ has been thus calculated, all other properties at station 3 follow by substitution. Again, it is necessary to include an iterative loop to ensure saturation conditions at station 3. That determines $\delta m_{v}$ and completes the model for the transition from station 2 to station 3 .

The transition from station 1 to station 3 of Figure 1(b) is now modelled as a sequence of $N$ small steps with equal volume increments, not pressure increments as for the BEE, each with two sub-steps:

(1) expansion without condensation, with constant entropy of the air-vapour component of the air-vapour-fog mixture; and

(2) condensation to saturation at constant volume as per equations (4.4)-(4.11).

For this expansion model to be valid, changes in the entropy of droplets are assumed to be negligible for sub-step 1, and changes in the internal energy of condensed droplets are assumed to be negligible for sub-step 2. This will be acceptable provided not too much condensed water is present, which is equivalent to assuming that the inlet temperature $T_{1}$ is not too high. Note that the constants $e_{1}, e_{2}$ and $e_{3}$ need to be updated throughout the expansion process. 


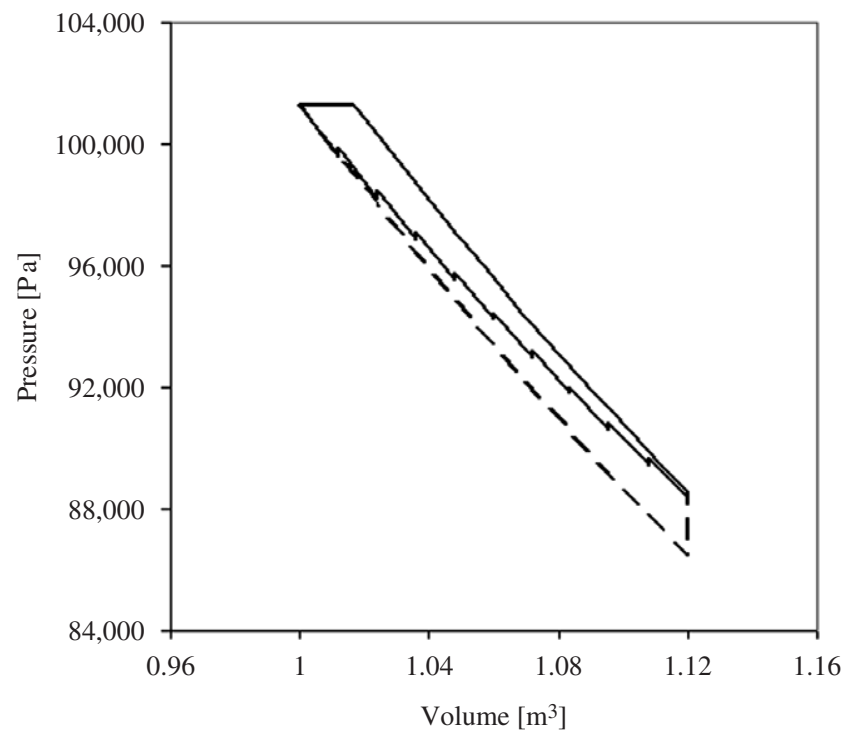

FIGURE 9. Effect of condensation during expansion is modelled by the sawtooth line: $T_{1}=20{ }^{\circ} \mathrm{C}$, $P_{a 1}=98961 \mathrm{~Pa}, P_{v 1}=2339 \mathrm{~Pa}$ (exactly at saturation), $r=1.12$. Only 10 steps are shown above, but 60 steps were used for the results of Section 5.

The expansion process is illustrated in Figure 9, in which only 10 steps are depicted. For the results presented in Section 5, 60 steps were used. Since no heat is added and condensation is assumed to be reversible, expansion from station 1 to station 3 is a constant-entropy process, which permits a good check on numerical results. The entropy of the air-vapour mixture at station 1 should equal the entropy of the airvapour-fog mixture at station 3 .

The remaining stage is compression from station 3 to station 4 . This involves identical equations to (2.20)-(2.25), but in which $\delta m_{v}$ is now negative. The compression ratio $\theta=V_{4} / V_{3}$ can be determined by matching the outlet pressure to the inlet pressure. The analysis proceeds as that for the BEE in Section 2 and gives

$$
T_{4}=T_{3} \theta^{-e_{4} / e_{5}}
$$

in which $e_{4}$ and $e_{5}$ are defined by

$$
\begin{aligned}
& e_{4}=\left(\gamma_{a}-1\right) m_{a} C_{a V}+\left(\gamma_{v}-1\right)\left(m_{v}+\delta m_{v}\right) C_{v V}, \\
& e_{5}=m_{a} C_{a V}+\left(m_{v}+\delta m_{v}\right) C_{v V},
\end{aligned}
$$

and $\theta$ by

$$
\theta=\left(\frac{P_{a 3}+P_{v 3}}{P_{a 1}+P_{v 1}}\right)^{e_{5} /\left(e_{4}+e_{5}\right)} .
$$

All quantities at station 4 can now be determined by substitution. It is noted again that $\delta m_{v}<0$ for the BDE heat pump. 
TABLE 2. State of the heat pump for the entropy check. The amount of water condensed during expansion is $0.01729-0.01262=0.00467 \mathrm{~kg}$.

\begin{tabular}{ccccccc}
\hline Station & $V\left(\mathrm{~m}^{3}\right)$ & $T\left({ }^{\circ} \mathrm{C}\right)$ & $m_{a}(\mathrm{~kg})$ & $m_{v}(\mathrm{~kg})$ & $P_{a}(\mathrm{~Pa})$ & $P_{v}(\mathrm{~Pa})$ \\
\hline 1 & 1.0 & 20 & 1.17623 & 0.01729 & 98961 & 2339 \\
3 & 1.2 & 11.6 & 1.17623 & 0.01262 & 80109 & 1382 \\
4 & 1.02706 & 29.8 & 1.17623 & 0.01262 & 99582 & 1718 \\
\hline
\end{tabular}

The net work output $W$ during the cycle is given by the integral

$$
W=\int_{V_{1}}^{V_{3}}\left(P_{a}+P_{v}-\left[P_{a 1}+P_{v 1}\right]\right) d V+\int_{V_{3}}^{V_{4}}\left(P_{a}+P_{v}-\left[P_{a 1}+P_{v 1}\right]\right) d V .
$$

The first term is negative and is the work required during expansion; this was evaluated numerically as for the BEE heat engine. The second term is positive and is the work received during compression; this was evaluated analytically. In fact, $W<0$, that is work is required to complete the cycle.

\section{Results for the BDE heat pump}

In the results presented in this section for the BDE heat pump, the inlet air was assumed to be fully saturated at the inlet temperature, which was between 5 and $25^{\circ} \mathrm{C}$. The initial volume was $V_{1}=1 \mathrm{~m}^{3}$ and the expansion ratio $r=V_{2} / V_{1}$ varied between 1.04 and 1.68. The inlet densities of air and vapour were given by the ideal gas laws $P=R \rho T$, where $R_{a}$ and $R_{v}$ had the same values as in Section 3. The specific heat capacities also had the same values as those used in Section 3. The latent heat of condensation at constant volume, $L$, was given by linear interpolation of tabulated values [4, Table A-4]. Results were again obtained using 60 steps for the transition from station 1 to station 3 .

A key requirement in the simulations was to determine saturation conditions for air-vapour mixtures. This is required for each of the small steps in the expansion phase between stations 1 and 3. At a particular temperature, saturation occurs when the partial vapour pressure $P_{v}$ reaches the saturation pressure given by the Clapeyron-Clausius equation [4, Section 11-3]. For the simulations presented below, the saturation vapour pressure was given by linear interpolation of tabulated values [4, Table A-4].

The entropy check anticipated in Section 4 is now described for the case $V_{1}=1 \mathrm{~m}^{3}$, $T_{1}=20{ }^{\circ} \mathrm{C}, P_{a 1}=98961 \mathrm{~Pa}, P_{v 1}=2339 \mathrm{~Pa}$ (i.e. at saturation) and $r=1.2$.

Based on linear interpolation of tabulated values (Table 2) of specific entropy for air and saturated water and vapour [4, Tables A-4 and A-17], the change in entropy of the air component of the air-vapour-fog mixture from station 1 to station 3 was $+37.12 \mathrm{~J} \mathrm{~K}^{-1}$, whilst the change in entropy of the vapour-fog component of the mixture was $-37.20 \mathrm{~J} \mathrm{~K}^{-1}$. (As noted in Section 3, the specific entropy of air as an ideal gas depends on both temperature and pressure; see [4, Equation (6-42)].) 

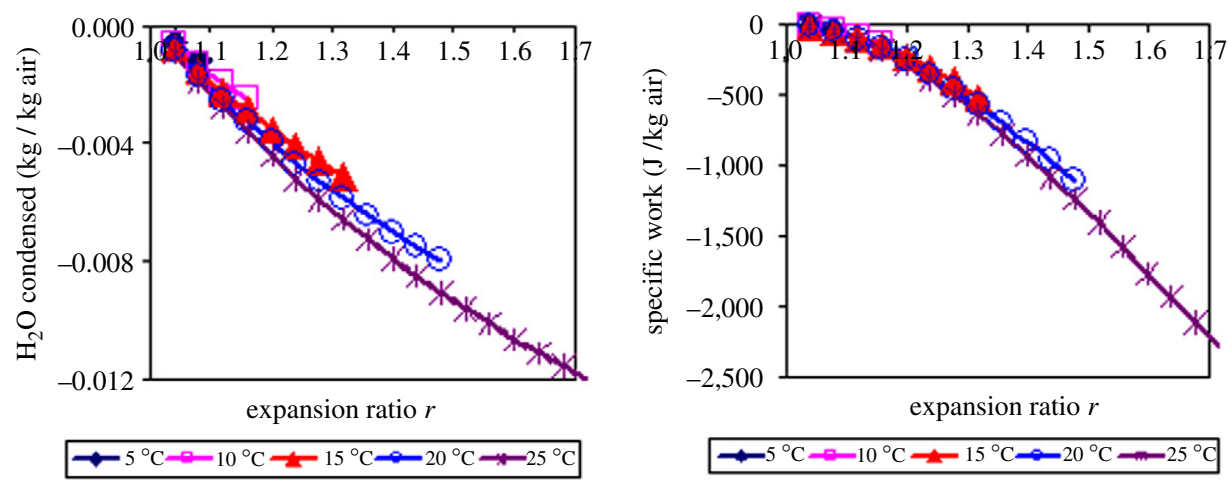

FIGURE 10. Left-hand figure: change in the mass of vapour $\delta m_{v}$ ( $\mathrm{kg}$ per $\mathrm{kg}$ of dry air; in fact $\delta m_{v}<0$, which confirms that water has condensed during the thermodynamic cycle). Right-hand figure: specific work output $w\left(\mathrm{~J} \mathrm{~kg}^{-1}\right.$; in fact $w<0$, which confirms that work is required to complete the thermodynamic cycle). Results are shown as a function of the expansion ratio $r$ for various values of inlet temperature $T_{1}$.

The entropy change of the entire mixture was $-0.08 \mathrm{~J} \mathrm{~K}^{-1}$, which is acceptably close to zero given the various approximations made (constant specific heats, ideal gas laws, finite number of expansion steps, linear interpolation of various quantities). The magnitude of the entropy change, given by addition of two large quantities of opposing sign, is 0.001 times the sum of the magnitudes of the individual quantities. This gives confidence in the computational procedure described in Section 4.

Figure 10 shows the change in mass of vapour and work output during the thermodynamic cycle of the BDE heat pump. Both quantities are negative, showing that water vapour has condensed and work is required to complete the cycle. This is a classic feature of heat pumps: heat is taken from a low-temperature reservoir (condensation at reduced pressure and low temperature) and provided to a reservoir at higher temperature (the exhaust). Work must be done during the cycle. The validity of the foregoing theory is limited in that the temperature at the coldest point, $T_{3}$, must be above freezing. If it were below freezing, then different values need to be used for the saturation vapour pressure. This limits the expansion ratio $r$, but only for application of the theory presented in Section 4. In practice, higher values of $r$ would be possible since vapour can freeze onto droplets with an ice coating. The saturation vapour pressure over ice is actually lower than that over water.

The coefficient of performance $C O P$ of the BDE heat pump is defined by

$$
C O P=\frac{m_{a} C_{a P}\left(T_{4}-T_{1}\right)}{W},
$$

where $W$ is given by equation (4.16). Figure 11 shows the back-work ratio (expansion work divided by compression work) and $C O P$. The back-work ratio is always greater than one (work must be supplied!) and increases as the inlet temperature $T_{1}$ increases. Wiggles in the back-work plots are artefacts of the computational process, particularly 

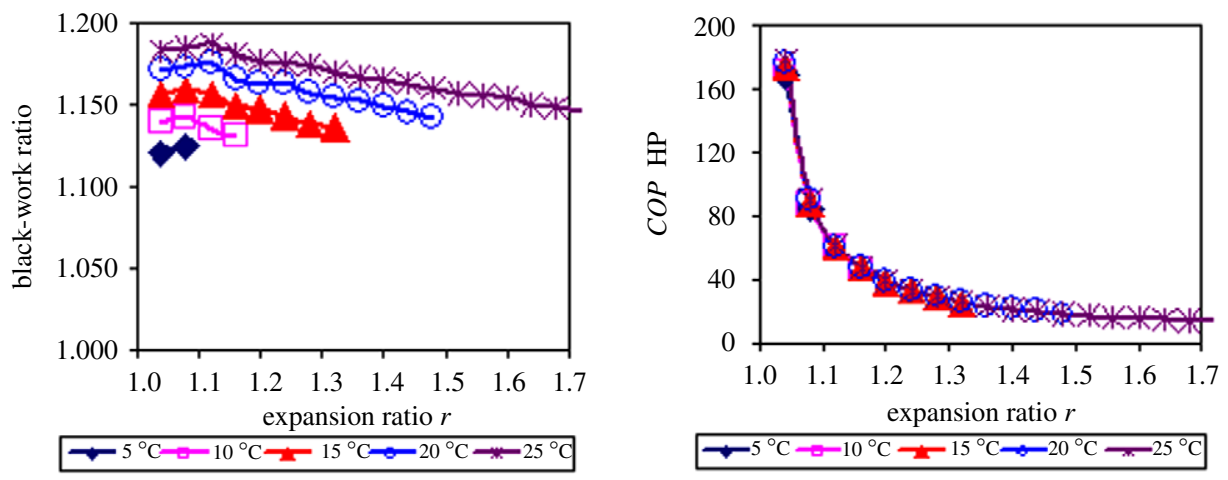

FIGURE 11. Left-hand figure: back-work ratio (expansion work divided by compression work). Righthand figure: coefficient of performance, COP, as defined by equation (63). Results are shown as a function of the expansion ratio $r$ for various values of inlet temperature $T_{1}$.
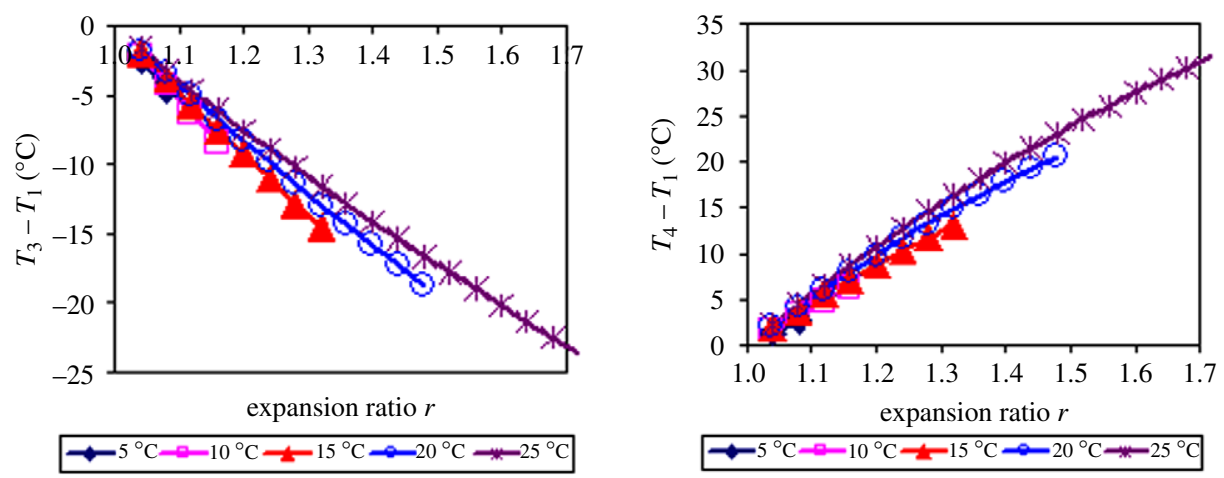

FIgURE 12. Left-hand figure: temperature change $T_{3}-T_{1}\left({ }^{\circ} \mathrm{C}\right)$ from the inlet to the coldest point (station 3). Right-hand figure: temperature change $T_{4}-T_{1}\left({ }^{\circ} \mathrm{C}\right)$ from inlet to outlet. Results are shown as a function of the expansion ratio $r$ for various values of inlet temperature $T_{1}$.

various linear interpolations between tabulated values. The results for the coefficient of performance, given by equation (5.1), are noteworthy. For example, for inlet temperatures of 10 and $15^{\circ} \mathrm{C}$ and maximum allowable values of the expansion ratio $r$, $C O P$ takes the values 47 and 26 respectively. The BDE is a very good heat pump!

Figure 12 shows temperature change $T_{3}-T_{1}$ from the inlet to the coldest point and the temperature change $T_{4}-T_{1}$ from inlet to outlet. (Recall that $T_{3}$ must be above freezing for the theory to hold, thus limiting the expansion ratio.) At the maximum theoretical values of the expansion ratio $r$ and inlet temperatures of 10 and $15^{\circ} \mathrm{C}$, the outlet temperature is 7 and $13{ }^{\circ} \mathrm{C}$ hotter respectively than the inlet temperature.

\section{Discussion and conclusions}

The inputs to the BEE heat engine are water plus unsaturated air; the outputs are work and air that is cooled and moistened. The simulations show that evaporative 
coolers can deliver useful power, and that the work output increases as the inlet temperature increases.

The amount of work obtained during a cycle of the BEE is limited by the amount of water that can be evaporated before saturation occurs. That fact, together with the high back-work ratios associated with gas cycle engines, means that a piston-incylinder configuration is strongly preferred over a continuous flow configuration (that is turbine, evaporator and extraction fan in series). Positive aspects of a piston-incylinder configuration are good control over leakage and friction, and tolerance to droplets in the air.

Water consumption in the BEE is a non-trivial issue. Small droplets are required for evaporation to occur quickly. Injection of droplets with diameter $15 \mu \mathrm{m}$ requires a water pump at approximately $5 \mathrm{MPa}$ pressure. For such a pump, the work required to inject $1 \mathrm{~g}$ of water is $5 \mathrm{~J}$. However, this is not particularly demanding considering the amount of work obtained. For example, with inlet temperature $T_{1}=100{ }^{\circ} \mathrm{C}$ and expansion ratio $r=1.8$, the specific power output is $7238 \mathrm{~J}$ per $\mathrm{kg}$ of air throughput and the specific water consumption is $0.0237 \mathrm{~kg}$ per $\mathrm{kg}$ air. To inject the water requires $1.6 \%$ of the theoretical work output of the engine. In a relative sense, both water injection and consumption become progressively less of a problem as the inlet temperature increases.

In general, water injected into the BEE will need to be free from solid matter and preferably from chemical impurities at well. If water containing salt or other dissolved substances is used for injection instead of pure water, dissolved substances will reduce the saturation vapour pressure according to Raoult's law [5] and thereby the work output per cycle. Deposition of chemical residues inside the engine should also be avoided.

Unpublished experimental work by the author shows that it takes a little more than a second for injected water droplets to disperse from a high-pressure nozzle and fill an 80 litre container. The additional time required for evaporation once the droplets have been dispersed is not expected to be particularly large (unpublished calculations by the author, confirmed by Sirignano [7]). The cycle time for the BEE will be more than one second-but perhaps not much more, depending on how the expansion and compression steps are managed. In fact, an inertial double-acting piston-in-cylinder configuration is anticipated to require approximately three seconds for two complete cycles. Such a configuration is planned for the first experimental BEE, currently under construction.

Possible applications for the BEE heat engine include efficient evaporative coolers, power generation in arid climates using solar pre-heating, and power generation using industrial waste heat, especially the exhaust of open-cycle gas turbines (OCGTs). Other applications are expected to be discovered.

The inputs to the BDE heat pump are cool moist air plus power; the outputs are condensed water plus air that is warmed and dried. The main application is anticipated to be for space heating, although dryness of the output air might be important for certain applications. Since condensed droplets are collected during the BDE cycle, the 
BDE would also 'wash' the air, which might be relevant if the ambient atmosphere contains unwanted particles.

The BEE and BDE will be slow-revving. Also, since the BEE and BDE devices are based on expansion, not compression, they are expected to be large compared to automobile or aircraft engines of comparable power output. The large size will help minimise some of the inevitable practical losses (see below). With careful design, an increase in the volumetric capacity is not expected to have a strong effect on the cycle time. Large slow-revving devices will probably not be suitable for mobile applications (except perhaps for boats or trains) and are certainly unconventional, but still might be economically advantageous for stationary applications.

Comments in the previous paragraph are now illustrated by an example in which the intake air for the BEE is sourced from the exhaust of an open-cycle gas turbine at $500^{\circ} \mathrm{C}$ and partial pressures $P_{a 1}=92.5 \mathrm{kPa}$ and $P_{v 1}=8.8 \mathrm{kPa}$. The density of dry air at the inlet is $\rho_{a 1}=0.414 \mathrm{~kg} \mathrm{~m}^{-1}$. The theory of Section 2 predicts that the specific power output of the BEE is $148 \mathrm{~kJ}$ (kg dry air throughput) ${ }^{-1}$ at an expansion ratio $r=4.0$. Current thoughts on the construction of the BEE are that it would have an inertial double-acting piston-in-cylinder configuration. If the engine completes two power strokes in three seconds, as is currently thought achievable, the theoretical average power output per unit volume would be $9.3 \mathrm{~kW} \mathrm{~m}^{-3}$. That is expected to be similar to the power output per unit volume of a steam turbine to use the OCGT exhaust, bearing in mind the need for high-pressure boiler and condenser. Note that the BEE does not need a boiler or condenser, thereby saving on capital costs.

The theory and results presented above do not include the effects of various losses, in particular:

- losses associated with flow through inlet and outlet valves;

- leakage around the piston (or if leakage is absent, then friction);

- mechanical friction in the mechanism used to extract or supply power;

- losses associated with the high-back work ratio;

- heat transfer through the cylinder walls;

- energy required for water treatment;

- energy required to inject water droplets between stations 2 and 3 for the BEE; and

- energy required to collect condensed water at station 3 in the BDE; collection is anticipated to be carried out by sweeping a secondary piston with cyclones through the chamber at the point of maximum expansion.

An experimental BEE is currently under construction. Experimental investigation of losses will be an important task once construction of the engine is complete.

\section{Acknowledgements}

The author gratefully acknowledges helpful input to earlier work by Andrew Barton, Christopher Barton, Neville Fowkes and Colin Please. Anthony Kitchener is thanked for contributions to recent work. This paper has been significantly improved as a result of comments by anonymous referees. 


\section{References}

[1] N. G. Barton, "Thermodynamic model for a two-stroke evaporation engine", Proc. Australian and New Zealand Solar Energy Society, Canberra, 2006.

[2] N. G. Barton, "The Bernoulli evaporation turbine and Bernoulli condensation heat pump", unpublished manuscript available from the author.

[3] G. K. Batchelor, An introduction to fluid dynamics (Cambridge University Press, Cambridge, 1967).

[4] Y. A. Çengel and M. A. Boles, Thermodynamics: an engineering approach, 2nd edn (McGraw-Hill, New York, 1994).

[5] D. A. McQuarrie and P. A. Rock, General chemistry, 3rd edn (W. H. Freeman, New York, 1991).

[6] F. Reif, Fundamentals of statistical and thermal physics (McGraw-Hill, New York, 1965).

[7] W. A. Sirignano, Fluid dynamics and transport of droplets and sprays (Cambridge University Press, Cambridge, 1999). 